Proyecciones Journal of Mathematics

Vol. 29, No 1, pp. 41-48, May 2010.

Universidad Católica del Norte

Antofagasta - Chile

\title{
MEASURES OF FUZZY SUBGROUPS
}

\author{
$S H U$ - RUI SHI \\ CAPITAL NORMAL UNIVERSITY, CHINA \\ Received : December 2009. Accepted : January 2010
}

\begin{abstract}
In this paper, we introduce the notion of degree to which a fuzzy subset is a fuzzy subgroup by means of the implication operator of $[0,1]$. A fuzzy subset $\mu$ in a group $G$ is a fuzzy subgroup if and only if its subgroup degree $m_{g}(\mu)=1$. Some properties of subgroup degrees are investigated.
\end{abstract}

Keywords : Fuzzy subgroup, implication operator, subgroup degree

Subjclass : [2000]03E72, 08A72, 20N25 


\section{Introduction}

In 1971, A. Rosenfeld introduced the concept of fuzzy groups [14]. In 1979, Anthony and Sherwood [4] redefined fuzzy groups. Since then many results in group theory has been generalized to fuzzy setting. Some properties of products, set products, unions, intersections, the homomorphic images and preimages of fuzzy subgroups are introduced (see $[1,2,3,6,8,9,10,13$, 15]). Some authors discussed the relations between the fuzzy subgroups and their level subgroups. V. N. Dixit, et al. gave characterizations of fuzzy conjugate subgroups and fuzzy characteristic subgroups by their level subgroups [8].

From the above results, we can know that a fuzzy set in a group $G$ is either a fuzzy subgroup or not. The philosophy is developing a technique with which we no longer merely decide whether a fuzzy subset is a fuzzy subgroup or not but with which we actually measure a degree to which it is a fuzzy subgroup.

In this paper, our aim is to introduce the notion of degree to which a fuzzy subset of a group $G$ is a fuzzy subgroup by means of the implication operator of $[0,1]$.

\section{Preliminaries}

Throughout this paper, $[0,1]^{X}$ (or $I^{X}$ ) denotes the set of all fuzzy sets on $X$. $a$ denotes the constant fuzzy sets on $X$ taking the value $a$. For $A \in[0,1]^{X}$ and $a \in[0,1]$, we can use the notations:

$$
A_{[a]}=\{x \in X: A(x) \geq a\}, \quad A_{(a)}=\{x \in X: A(x)>a\} .
$$

A function $T:[0,1] \times[0,1] \rightarrow[0,1]$ is called a triangular norm (shortly t-norm) if it satisfies, for all $x, y, z \in[0,1]$, the following conditions:

(T1) $T(x, y)=T(y, x)$,

(T2) $T(x, T(y, z))=T(T(x, y), z)$,

(T3) if $y \leq z$, then $T(x, y) \leq T(x, z)$, i.e., $T(x, \cdot)$ is increasing,

(T4) $T(x, 1)=x$.

A function $I_{T}:[0,1] \times[0,1] \rightarrow[0,1]$ is called an R-implication if there exists a t-norm $T$ such that

$$
I_{T}(x, y)=\sup \{t \in[0,1]: T(x, t) \leq y\}, \quad x, y \in[0,1] .
$$

$I_{T}$ is also called the residual of the t-norm $T$. 


\section{The subgroup degrees of $L$-fuzzy sets}

A fuzzy subset $\mu$ of a group $G$ is said to be a fuzzy subgroup of $G$ [14] if for any $x, y \in G, \mu\left(x y^{-1}\right) \geq \mu(x) \wedge \mu(y)$.

According to the above definition, we know that a fuzzy subset of a group $G$ is either a fuzzy subgroup or not.

If the degree of $a \leq b$ is defined by $I_{T}(a, b)$, then we can naturally introduce the notion of subgroup degree as follows:

Definition 3.1. Let $\mu$ be a fuzzy subset in a group $G$ and $T$ be a $t$-norm on $[0,1]$. The subgroup degree $m_{g}(\mu)$ of $\mu$ is defined as

$$
m_{g}(\mu)=\bigwedge_{x, y \in G} I_{T}\left(T(\mu(x), \mu(y)), \mu\left(x y^{-1}\right)\right) .
$$

If $T$ is taken as $T(a, b)=a \wedge b$ for any $a, b \in[0,1]$, then obviously $\mu$ is a fuzzy subgroup of $G$ if and only if $m_{g}(\mu)=1$.

In the sequel, we shall take $T$ as $T(a, b)=a \wedge b$ for any $a, b \in[0,1]$ and $I_{T}(a, b)$ is written as $a \mapsto b$.

Example 3.2. Let $\mathbf{Z}$ be an integer additive group. Define $\mu: \mathbf{Z} \rightarrow[0,1]$ by

$$
\mu(n)= \begin{cases}0.3, & \text { if } n \text { is even; } \\ 0.5, & \text { if } n \text { is odd }\end{cases}
$$

It is easy to check that $m_{g}(\mu)=0.3$.

Theorem 3.3. Let $\mu$ be a fuzzy subset in a group $G$. Then

$$
m_{g}(\mu)=\bigwedge_{x, y \in G}((\mu(x) \wedge \mu(y)) \mapsto \mu(x y)) \wedge \bigwedge_{x \in G}\left(\mu(x) \mapsto \mu\left(x^{-1}\right)\right) .
$$

Proof. It is obvious that

$$
\begin{aligned}
m_{g}(\mu) & =\bigwedge_{x, y \in G}\left((\mu(x) \wedge \mu(y)) \mapsto \mu\left(x y^{-1}\right)\right) \\
& \leq \bigwedge_{y \in G}\left((\mu(e) \wedge \mu(y)) \mapsto \mu\left(y^{-1}\right)\right) \wedge \bigwedge_{x \in G}(\mu(x) \wedge \mu(x) \mapsto \mu(e) \wedge \mu(x)) . \\
& \leq \bigwedge_{x \in G}\left(\mu(x) \mapsto \mu\left(x^{-1}\right)\right) .
\end{aligned}
$$


Further we have

$$
\begin{aligned}
m_{g}(\mu) & \leq \bigwedge_{x, y \in G}\left((\mu(x) \wedge \mu(y)) \mapsto \mu\left(x y^{-1}\right)\right) \wedge \bigwedge_{x \in G}\left(\mu(x) \mapsto \mu\left(x^{-1}\right)\right) \\
& \leq \bigwedge_{x, y \in G}\left((\mu(x) \wedge \mu(y)) \mapsto \mu\left(x y^{-1}\right)\right) \wedge \bigwedge_{x, y \in G}\left(\mu(x) \wedge \mu(y) \mapsto \mu(x) \wedge \mu\left(y^{-1}\right)\right) \\
& \leq \bigwedge_{x, y \in G}((\mu(x) \wedge \mu(y)) \mapsto \mu(x y)) .
\end{aligned}
$$

This shows

$$
m_{g}(\mu) \leq \bigwedge_{x, y \in G}((\mu(x) \wedge \mu(y)) \mapsto \mu(x y)) \wedge \bigwedge_{x \in G}\left(\mu(x) \mapsto \mu\left(x^{-1}\right)\right) .
$$

Moreover by

$$
\begin{aligned}
& \bigwedge_{x, y \in G}((\mu(x) \wedge \mu(y)) \mapsto \mu(x y)) \wedge \bigwedge_{x \in G}\left(\mu(x) \mapsto \mu\left(x^{-1}\right)\right) \\
\leq & \bigwedge_{x, y \in G}((\mu(x) \wedge \mu(y)) \mapsto \mu(x y)) \wedge \bigwedge_{x, y \in G}\left(\mu(x) \wedge \mu(y) \mapsto \mu(x) \wedge \mu\left(y^{-1}\right)\right) \\
\leq & \bigwedge_{x, y \in G}\left((\mu(x) \wedge \mu(y)) \mapsto \mu\left(x y^{-1}\right)\right)=m_{g}(\mu)
\end{aligned}
$$

we obtain

$$
m_{g}(\mu)=\bigwedge_{x, y \in G}((\mu(x) \wedge \mu(y)) \mapsto \mu(x y)) \wedge \bigwedge_{x \in G}\left(\mu(x) \mapsto \mu\left(x^{-1}\right)\right) .
$$

The following lemma is obvious.

Lemma 3.4. Let $\mu$ be a fuzzy subset in a group $G$. Then $m_{g}(\mu) \geq a$ if and only if for any $x, y \in G$,

$$
\mu(x) \wedge \mu(y) \wedge a \leq \mu\left(x y^{-1}\right) .
$$

By Lemma 3.4, we can easily obtain the following result.

Theorem 3.5. Let $\mu$ be a fuzzy subset in a group $G$. Then

$$
m_{g}(\mu)=\bigvee\left\{a \in(0,1]: \mu(x) \wedge \mu(y) \wedge a \leq \mu\left(x y^{-1}\right), \forall x, y \in G\right\} .
$$

Theorem 3.6. Let $\mu$ be a fuzzy subset in a group $G$. Then

$$
m_{g}(\mu)=\bigvee\left\{a \in(0,1]: \forall b \in(0, a], \mu_{[b]} \text { is a subgroup of } G\right\} .
$$


Proof. Suppose that $\mu(x) \wedge \mu(y) \wedge a \leq \mu\left(x y^{-1}\right), \forall x, y \in G$. Then for any $b \in(0, a]$ and for any $x, y \in \mu_{[b]}$, we have

$$
\mu\left(x y^{-1}\right) \geq \mu(x) \wedge \mu(y) \wedge a \geq \mu(x) \wedge \mu(y) \wedge b=\mu(x) \wedge \mu(y) \geq b,
$$

this shows $x y^{-1} \in \mu_{[b]}$. Therefore $\mu_{[b]}$ is a subgroup of $G$. Hence

$$
\begin{aligned}
m_{g}(\mu) & =\bigvee\left\{a \in(0,1]: \mu(x) \wedge \mu(y) \wedge a \leq \mu\left(x y^{-1}\right), \forall x, y \in G\right\} \\
& \leq \bigvee\left\{a \in(0,1]: \forall b \in(0, a], \mu_{[b]} \text { is a subgroup of } G\right\} .
\end{aligned}
$$

Conversely, assume that $a \in(0,1]$ and $\forall b \in(0, a], \mu_{[b]}$ is a subgroup of $G$. For any $x, y \in G$, let $b=\mu(x) \wedge \mu(y) \wedge a$, then $b \leq a$ and $x, y \in \mu_{[b]}$, thus $x y^{-1} \in \mu_{[b]}$, i.e. $\mu\left(x y^{-1}\right) \geq b=\mu(x) \wedge \mu(y) \wedge a$. This means that

$$
\begin{aligned}
m_{g}(\mu) & =\bigvee\left\{a \in(0,1]: \mu(x) \wedge \mu(y) \wedge a \leq \mu\left(x y^{-1}\right), \forall x, y \in G\right\} \\
& \geq \bigvee\left\{a \in(0,1]: \forall b \leq a, \mu_{[a]} \text { is a subgroup of } G\right\} .
\end{aligned}
$$

Theorem 3.7. Let $\mu$ be a fuzzy subset in a group $G$. Then

$$
m_{g}(\mu)=\bigvee\left\{a \in(0,1]: \forall b<a, \mu_{(b)} \text { is a subgroup of } G\right\} \text {. }
$$

Proof. Suppose that $a \in(0,1]$ and $\mu(x) \wedge \mu(y) \wedge a \leq \mu\left(x y^{-1}\right)$ for any $x, y \in G$. Let $b<a$, and $x, y \in \mu_{(b)}$. Then $b<\mu(x) \wedge \mu(y) \wedge a \leq \mu\left(x y^{-1}\right)$. Hence $x y^{-1} \in \mu_{(b)}$. This shows that $\mu_{(b)}$ is a subgroup of $G$. Therefore

$$
\begin{aligned}
m_{g}(\mu) & =\bigvee\left\{a \in(0,1]: \mu(x) \wedge \mu(y) \wedge a \leq \mu\left(x y^{-1}\right), \forall x, y \in G\right\} \\
& \leq \bigvee\left\{a \in(0,1]: \forall b<a, \mu_{(b)} \text { is a subgroup of } G\right\} .
\end{aligned}
$$

Conversely, assume that $a \in\left\{a \in(0,1]: \forall b<a, \mu_{(b)}\right.$ is a subgroup of $G$ \}. Now we prove that $\mu(x) \wedge \mu(y) \wedge a \leq \mu\left(x y^{-1}\right)$ for any $x, y \in G$. Let $b<\mu(x) \wedge \mu(y) \wedge a$. Then $\mu(x)>b, \mu(y)>b$ and $a>b$, i.e., $x, y \in \mu_{(b)}$. Since $\mu_{(b)}$ is a subgroup of $G$, it holds that $x y^{-1} \in \mu_{(b)}$, i.e. $\mu\left(x y^{-1}\right)>b$. This shows that $\mu(x) \wedge \mu(y) \wedge a \leq \mu\left(x y^{-1}\right)$. Therefore

$$
\begin{aligned}
m_{g}(\mu) & =\bigvee\left\{a \in(0,1]: \mu(x) \wedge \mu(y) \wedge a \leq \mu\left(x y^{-1}\right), \forall x, y \in G\right\} \\
& \geq \bigvee\left\{a \in(0,1]: \forall b<a, \mu_{(b)} \text { is a subgroup of } G\right\} .
\end{aligned}
$$

Theorem 3.8. Let $\left\{\mu_{i}\right\}_{i \in I}$ be a family of fuzzy subsets in a group $G$. Then $m_{g}\left(\bigwedge_{i \in I} \mu_{i}\right) \geq \bigwedge_{i \in I} m_{g}\left(\mu_{i}\right)$. 
Proof. This can be proved from the following inequality.

$$
\begin{aligned}
m_{g}\left(\bigwedge_{i \in I} \mu_{i}\right) & \left.=\bigwedge_{x, y \in G}\left(\left(\bigwedge_{i \in I} \mu_{i}\right)(x) \wedge\left(\bigwedge_{i \in I} \mu_{i}\right)(y)\right) \mapsto\left(\bigwedge_{i \in I} \mu_{i}\right)\left(x y^{-1}\right)\right) \\
& \left.=\bigwedge_{x, y \in G} \bigwedge_{i \in I}\left(\left(\bigwedge_{i \in I} \mu_{i}\right)(x) \wedge\left(\bigwedge_{i \in I} \mu_{i}\right)(y)\right) \mapsto \mu_{i}\left(x y^{-1}\right)\right) \\
& \geq \bigwedge_{x, y \in G} \bigwedge_{i \in I}\left(\left(\mu_{i}(x) \wedge \mu_{i}(y)\right) \mapsto \mu_{i}\left(x y^{-1}\right)\right) \\
& =\bigwedge_{i \in I} \bigwedge_{x, y \in G}\left(\left(\mu_{i}(x) \wedge \mu_{i}(y)\right) \mapsto \mu_{i}\left(x y^{-1}\right)\right) \\
& =\bigwedge_{i \in I} m_{g}\left(\mu_{i}\right) .
\end{aligned}
$$

The following result is obvious.

Theorem 3.9. Let $f$ be a homomorphism from a group $G$ to a group $G^{\prime}$.

(1) If $\mu$ is a fuzzy subset in $G$, then $m_{g}(f \rightarrow(\mu)) \geq m_{g}(\mu)$, where $f^{\rightarrow}(\mu)(y)=$ $\bigvee\{\mu(x): f(x)=y\}$;

(2) If $\eta$ is a fuzzy subset in $G^{\prime}$, then $m_{g}\left(f^{\leftarrow}(\eta)\right) \geq m(\eta)$, where $f^{\leftarrow}(\eta)(x)=$ $\eta(f(x))$.

Proof. (1) can be proved from Theorem 3.5 and the following fact.

$$
\begin{aligned}
& m_{g}(f \rightarrow(\mu)) \\
= & \bigvee\left\{a \in(0,1]: f \rightarrow(\mu)\left(x^{\prime}\right) \wedge f \rightarrow(\mu)\left(y^{\prime}\right) \wedge a \leq f \rightarrow(\mu)\left(x^{\prime} y^{\prime-1}\right), \forall x^{\prime}, y^{\prime} \in G^{\prime}\right\} \\
= & \bigvee\left\{a \in(0,1]: \bigvee_{f(x)=x^{\prime}} \mu(x) \wedge \underset{f(y)=y^{\prime}}{\bigvee} \mu(y) \wedge a \leq \underset{f(z)=x^{\prime} y^{\prime-1}}{\bigvee} \mu(z), \forall x^{\prime}, y^{\prime} \in G^{\prime}\right\} \\
\geq & \bigvee\left\{a \in(0,1]: \mu(x) \wedge \mu(y) \wedge a \leq \mu\left(x y^{-1}\right), \forall x, y \in G\right\} \\
= & m_{g}(\mu) .
\end{aligned}
$$

(2) can be proved from the following fact.

$$
\begin{aligned}
m_{g}\left(f^{\leftarrow}(\eta)\right) & =\bigwedge_{x, y \in G}\left(\left(f^{\leftarrow}(\eta)(x) \wedge f^{\leftarrow}(\eta)(y)\right) \mapsto f^{\leftarrow}(\eta)\left(x y^{-1}\right)\right) \\
& =\bigwedge_{x, y \in G}\left((\eta(f(x)) \wedge \eta(f(y))) \mapsto \eta\left(f(x) f(y)^{-1}\right)\right) \\
& \geq \bigwedge_{x^{\prime}, y^{\prime} \in G^{\prime}}\left(\left(\eta\left(x^{\prime}\right) \wedge \eta\left(y^{\prime}\right)\right) \mapsto \eta\left(x^{\prime} y^{\prime-1}\right)\right) \\
& =m_{g}(\eta) .
\end{aligned}
$$




\section{References}

[1] N. Ajmal, Homomorphism of fuzzy groups, Correspondence theorem and fuzzy quotient groups, Fuzzy Sets and Systems 61, no. 3, pp. 329$337,(1994)$.

[2] N. Ajmal, Fuzzy groups with sup property, Information Sciences 93, no. 3, pp. 247-264, (1996).

[3] N. Ajmal, Fuzzy group theory: A comparison of different notions of product of fuzzy sets, Fuzzy Sets and Systems 110, no. 3, pp. 437-446, (2000).

[4] J.M. Anthony and H. Sherwood, Fuzzy groups redefined, J. Math. Anal. Appl. 69, no. 1, pp. 124-130, (1979).

[5] J.M. Anthony and H. Sherwood, A characterization of fuzzy subgroups, Fuzzy Sets and Systems 7, no. 3, pp. 297-305, (1982).

[6] I. Chon, Fuzzy subgroups as products, Fuzzy Sets and Systems 141, no. 3, pp. 505-508, (2004).

[7] P.S. Das, Fuzzy groups and level subgroups, J. Math. Anal. Appl. 84, no. 1, pp. 264-269, (1981).

[8] V.N. Dixit et al., Level subgroups and union of fuzzy subgroups, Fuzzy Sets and Systems 37, no. 3, pp. 359-371, (1990).

[9] V.N. Dixit et al., Union of fuzzy subgroups, Fuzzy Sets and Systems 78, no. 1, pp. 121-123, (1996).

[10] M.S. Eroğlu, The homomorphic image of a fuzzy subgroup is always a fuzzy subgroup, Fuzzy Sets and Systems 33, no. 2, pp. 255-256, (1989).

[11] W.J. Liu, Fuzzy invariant subgroups and fuzzy ideals, Fuzzy Sets and Systems 8, no. 2, pp. 133-139, (1982).

[12] N.P. Mukherjee and P. Bhattacharya, Fuzzy normal subgroups and fuzzy cosets, Information Sciences 34, no. 3, pp. 225-239, (1984).

[13] A.K. Ray, On product of fuzzy subgroups, Fuzzy Sets and Systems 105, no. 1, pp. 181-183, (1999). 
[14] A. Rosenfeld, Fuzzy groups, J. Math. Anal. Appl. 35, no. 3, pp. 512517, (1971).

[15] H. Sherwood, Products of fuzzy subgroups, Fuzzy Sets and Systems 11, no. $1,79-89,(1983)$.

[16] W.-M. Wu, Normal fuzzy subgroups, Fuzzy Math. 1, no. 1, pp. 21-30, (1981).(in Chinese)

[17] Y. Zhang, Some properties on fuzzy subgroups, Fuzzy Sets and Systems 119, no. 3, pp. 427-438, (2001).

\section{Shu-Rui Shi}

School of Mathematics

Capital Normal University Beijing 100048

P. R. China

China

e-mail : shishurui341@126.com 